\title{
Einstein, Poincaré and Special Relativity
}

\author{
Emma Sallent Del Colombo
}

Published online: 16 May 2013

(C) Centro P.RI.ST.EM, Università Commerciale Luigi Bocconi 2013

\begin{abstract}
We present some considerations on the contributions of Henri Poincaré and Albert Einstein to Special Relativity, based on the 2004 paper by Olivier Darrigol, "The Mystery of the Einstein-Poincaré Connection".
\end{abstract}

Keywords Special relativity - Albert Einstein $\cdot$ Henri Poincaré

Albert Einstein is usually held to be the sole author of the theory of relativity. However, this belief has been questioned, especially with regard to the contributions of Henri Poincaré. The dearth of documents directly related to this subject, and the lack of cross-references between the two authors-who did actually know and speak to each other, but neither of whom ever cited the other-has made it even more difficult to shed light on this. The controversy, which is often based more on opinions than on facts, has sometimes led to judgments that have overtones that are nationalistic, anti-Semitic, or dictated by esprit d'école.

In 1905 Albert Einstein published four fundamental works on quantum theory, one on Brownian motion and two on relativity, including the article which is considered to be foundational for the special (or restricted) theory of relativity: "Zur Elektrodynamik bewegter Körper" [2].

Of the numerous papers were published during the centenary of so-called Annus mirabilis, of particular interest is that by the historian of physics Olivier Darrigol who, on the basis of the publications of the last 20 years, carried out a profound, critical and balanced examination

E. Sallent Del Colombo $(\bowtie)$

Departament de Física Fonamental, Universitat de Barcelona,

Martí i Franquès, 1, 08028 Barcelona, Spain

e-mail: emma.sallent@ub.edu of the relationship between the works of Einstein and Poincaré [1]. According to him, it is necessary to abandon the prejudice of considering the contributions of Einstein's contemporaries who dealt with relativity as being a priori inferior to those of Einstein himself, and instead evaluate whether they are correct or erroneous using Einstein as the measure. Darrigol shows that it is possible to carry out a much more interesting study that explains the similarities and differences between different approaches when pointless and sterile controversies over priority are abandoned.

In order to be able to compare and contrast the theories of Einstein and Poincare it is necessary to identify the conceptual stratum that they share by reading their individual contributions to each subject in their proper context. What are some of the ideas that they two theories have in common?

The analysis of the problems relating to the electrodynamics of bodies in motion led both Einstein and Poincaré in 1905 to postulate the validity of the principle of relativity. One formulation that the two had in common might be that the result of any conceivable experiment in physics is independent of the inertial frame of reference in which it is performed. Both assumed that the speed of light is the same if measured in different inertial frames of reference, and that space and time measured by observers belonging to different inertial frames of reference are related to each other by means of Lorentz transformations (generalisations of Galilean transformations). Both recognised that the Maxwell-Lorentz equations of electrodynamics are invariant with respect to these transformations. Finally, considered it requisite that the laws of physics be necessarily invariant with respect to the Lorentz transformations, and derived relativistic laws of motion.

On some points, such as the principle of relativity or the physical interpretation of the Lorentz transformations, 
Poincaré's contributions preceded by at least 5 years those of Einstein's published in 1905. On the other hand, many of their contributions were practically simultaneous. In 1905 Poincaré published an abridged version of his "Sur la dynamique de l'électron" [3] (which preceded the work of Einstein); the expanded version of the article appeared in 1906 [4].

What are the conceptual differences? According to Darrigol,

Einstein completely eliminated the ether, required that the expression of the laws of physics should be the same in any inertial frame, and introduced a "new kinematics" in which the space and time measured in different inertial systems were all on exactly the same footing. In contrast, Poincaré maintained the ether as a privileged frame of reference in which "true" space and time were defined, while he regarded the space and time measured in other frames as only "apparent." ...Einstein derived the expression of the Lorentz transformation from his two postulates (the relativity principle and the constancy of the velocity of light in a given inertial system), whereas Poincaré obtained these transformations as those that leave the MaxwellLorentz equations invariant [1].

These are conceptual differences that have no actual experimental consequences as far as electromagnetism and optics are concerned. As Lorentz commented, the difference is purely epistemological: it concerns the number of conventional and arbitrary elements that one wishes to introduce in the definitions of the basic physical concepts.

Are we then dealing with a case of simultaneous discovery? Could the same factors and same interests have contributed to producing similar studies, or did one of the two-chiefly Einstein-find some kind of inspiration in the work of the other? Perhaps it is possible to answer both questions in the affirmative.

Einstein had read Poincaré. But what exactly did he read? While it is not known for certain that he knew Poincaré's papers of 1905-1906, it is known that between 1902 and 1905 he read La Science et l'Hypothèse. Einstein, together with his friends Maurice Solovine and Conrad Habicht, founded the Akademie Olympia, an informal group that met to read and discuss books of physics and philosophy. Poincaré's book was the object of their rapt attention for several weeks! Some of the ideas that Einstein might have come across there are scepticism with regard to the existence of the ether, the principle of relativity, problems connected to the measure of time, and the problematic nature of the concept of the simultaneity of two events that take place in different places. All of these are ideas that then appeared in Einstein's 1905 paper.

As Darrigol suggests, it seems wiser to concede that Lorentz, Poincaré and Einstein all contributed to the emergence of the theory of relativity, that Poincaré and Einstein offered two different versions of the theory, and that Einstein gave form to what today is considered the best one. What then aided the establishment and consolidation of Einstein's point of view was the premature death of Poincaré, who Einstein outlived by no fewer than 43 years.

\section{References}

1. Darrigol, O.: The mystery of the Einstein-Poincaré connection. Isis 95, 614-626 (2004)

2. Einstein, A.: Zur Elektrodynamik bewegter Körper. Ann. Phys. 17(1905), 891-921 (1905)

3. Poincaré, H.: Sur la dynamique de l'électron. Comptes Rendues de l'Académie des Sciences 140, 1504-1508 (1905)

4. Poincaré, H.: Sur la dynamique de l'électron. Rendiconti del Circolo Matematico di Palermo 21, 129-176 (1906)

\section{Author Biography}

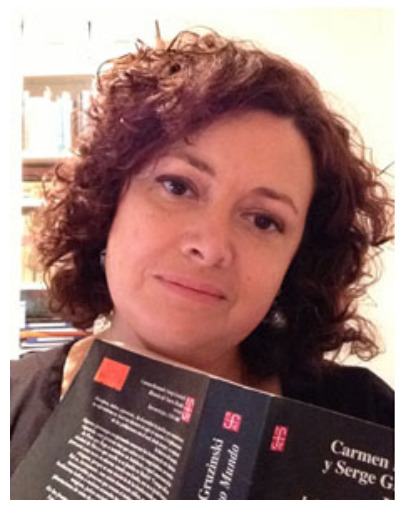

Emma Sallent is Professora Lectora in the Department of Física Fonamental at the University of Barcelona, and Vice President of the Societat Catalana d'Història de la Ciència $i$ de la Tècnica. Her research concerns the history of vector calculus and mathematical physics in Italy, as well as the history of science in Catalonia in the early twentieth century. In collaboration with Pietro Nastasi she is editing the correspondence between Gösta

Mittag-Leffler and Vito Volterra. With José Pardo Tomás she is working on Ulisse Aldrovandi's relationship to Spain, as part of the project entitled Cultura médica novohispana: circulación atlántica, recepción y apropiaciones. 